\title{
An Exact Closed-Form Expression for the BER of Binary Modulations with Dual-Branch Selection over Generalized- $K$ Fading
}

\author{
Imran Shafique Ansari*, Saad Al-Ahmadi**, Ferkan Yilmaz*, Mohamed-Slim Alouini*, and Halim Yanikomeroglu*** \\ * King Abdullah University of Science and Technology, Thuwal, Makkah Province, Saudi Arabia \\ Emails: \{imran.ansari, ferkan.yilmaz, slim.alouini\}@kaust.edu.sa \\ ** King Fahd University of Petroleum and Minerals, Dhahran, Eastern Province, Saudi Arabia \\ Email: ahmadisa@kfupm.edu.sa \\ *** Carleton University, Ottawa, Ontario, Canada \\ Email: halim@sce.carleton.ca
}

\begin{abstract}
Error performance is one of the main performance measures and the derivation of its closed-form expression has proved to be quite involved for certain systems. In this paper, a unified closed-form expression, applicable to different binary modulation schemes, for the bit error rate of dual-branch selection diversity based systems undergoing independent but not necessarily identically distributed generalized- $K$ fading is derived in terms of the extended generalized bivariate Meijer $G$-function.
\end{abstract}

\section{INTRODUCTION}

In recent times, different diversity schemes have taken up an important position in the wireless communication systems. The main reason behind this is that these different diversity schemes allow for multiple transmission and/or reception paths for the same signal [1]. One of the simplest diversity combining scheme is the selection combining (SC) diversity scheme where only one of the diversity branches is processed. Specifically, SC scheme chooses the branch with highest signal-to-noise ratio (SNR) [2]-[3].

Additionally, wireless communications are driven by a complicated phenomenon known as radio-wave propagation that is characterized by various effects including multipath fading, shadowing and path loss. The statistical behavior of these effects is described by different models depending on the nature of the communication environment. It is becoming necessary to study such effects i.e. large-scale fading as well as small-scale fading concurrently as the multihop relay networks are emerging in the current times. Using the Nakagami multipath fading model that is versatile enough to model various multipath fading conditions ranging from severe fading to nonfading scenario, and the Gamma model for shadowing has led to the generalized- $K$ (Gamma-Gamma) composite fading model [4]-[8]. Generalized- $K$ (GK) distribution, earlier used in radar applications and recently being used in the context of wireless digital communications over fading channels, is one of the relatively new tractable models used to describe the statistical behavior of multipath fading and shadowing effects as compared to log-normal based models. The GK fading model is quite general model as it includes $K$-distribution as its special case and accurately approximates many other fading models such as Nakagami- $m$ and Rayleigh-Lognormal (R-L) ([6] and references therein).

It is noteworthy to mention that bit error rate (BER) is one of the most important performance measures that forms the basis in designing wireless communication systems. Based on the open technical literature and up to the best of our knowledge, error analysis has been performed for dual diversity with SC over log-normal fading channels in closed-form using moment generating function (MGF) based approach in [9] and with Weibull fading channel as an approximate using characteristic function (CF) based approach in [10]. Additionally, error performance analysis of SC systems with independent and identically distributed (i.i.d.) GK fading branches was performed in [11] involving integral form expressions. Further, in [12] the analysis was performed for dual-branch SC citing the difficulty in deriving the expression for the probability density function (PDF). This issue was tackled in [13] for an arbitrary number of branches and the authors therein have described and utilized a method to perform the BER analysis directly from the cumulative density function (CDF) eliminating the need of deriving the PDF and relying on the Gauss-Laguerre quadrature technique.

In this work, we revisit this problem under the umbrella of the Fox $H$-functions and derive exact closed-form expression of the BER of binary modulation systems with dual-branch SC scheme and undergoing GK fading where the channels are independent but not necessarily identically distributed (i.n.i.d.). The remainder of the paper is organized as follows. Section II introduces the system and the GK channel model. 
Next, section III presents some statistical characteristics of GK fading channel model followed by the analytical BER analysis in section IV, and finally, section $\mathrm{V}$ discusses the results followed by summary the paper in section VI.

\section{The Generalized- $K$ Fading System and Channel MODEL}

A SC based communication system with a source and a destination is considered with i.n.i.d. channels as follows

$$
Y=\alpha X+n,
$$

where $Y$ is the received signal at the receiver end, $X$ is the transmitted signal, $\alpha$ is the channel gain, and $n$ is the additive white Gaussian noise (AWGN). In a Nakagami multipath fading channel, $\gamma=|\alpha|^{2}$ follows Gamma distribution; additionally, the shadowing component is also assumed to follow a Gamma distribution. Hence, the channel gains experience composite fading whose statistics follow a generalized- $K$ distribution given by

$$
p_{\gamma}(\gamma)=\frac{2 b^{m_{m}+m_{s}}}{\Gamma\left(m_{m}\right) \Gamma\left(m_{s}\right)} \gamma^{\frac{m_{m}+m_{s}}{2}-1} K_{m_{s}-m_{m}}(2 b \sqrt{\gamma}),
$$

where $\Gamma(\cdot)$ is the Gamma function as defined in [14, Eq. (8.310)], $m_{m}$ and $m_{s}$ are the Nakagami multipath fading and shadowing parameters, respectively. In (2) $K_{m}(\cdot)$ is the modified Bessel function of the second kind and order $m$, $b=\sqrt{\frac{m_{m} m_{s}}{\Omega_{0}}}$, and $\Omega_{0}$ is the mean of the local power. The parameters $m_{m}$ and $m_{s}$ quantify the severity of multipath fading and shadowing, respectively, in the sense that small values of $m_{m}$ and $m_{s}$ indicate severe multipath fading and shadowing conditions respectively, and vice versa. The instantaneous SNR of the $n^{t h}$ branch is given by $\gamma_{n}=\left(E_{b} / N_{0}\right) x_{n}{ }^{2}$ where $x_{n}$ is the signal amplitude for the $n^{\text {th }}$ branch, $E_{b}$ is the average energy per bit and $N_{0}$ is the power spectral density of the AWGN.

\section{Statistical Characteristics}

The PDF and CDF expressions of the GK random variables (RVs) can also be written in terms of Meijer $G$-function [14] as shown in the lemma given below.

Lemma: The PDF of a GK RV can be expressed in terms of Meijer- $G$ function as

$$
\begin{aligned}
& p_{\gamma}(y)=\left(\frac{m_{m} m_{s}}{\Gamma\left(m_{m}\right) \Gamma\left(m_{s}\right) \Omega_{0}}\right) \\
& \times G_{0,2}^{2,0}\left[\left(\frac{m_{m} m_{s}}{\Omega_{0}}\right) y \mid m_{m}-1, m_{s}-1\right], y>0 .
\end{aligned}
$$

Proof: We may use the fact that the PDF of the product of $N$ independent Gamma RVs can expressed as a $H$-function PDF that is given by [15, Eq. (6.4.9)] as follows

$$
\begin{aligned}
& p(x)=\left(\prod_{i=1}^{N} \frac{1}{\theta_{i} \Gamma\left(k_{i}\right)}\right) \\
& \times H_{0, N}^{N, 0}\left[\left(\prod_{1}^{N} \frac{1}{\theta_{i}}\right) x \mid\left(k_{i}-1,1\right), \ldots,\left(k_{N}-1,1\right)\right], x>0 .
\end{aligned}
$$

Then, with $N=2$, the GK PDF can be expressed as

$$
\begin{aligned}
& p(x)=\left(\frac{m_{m} m_{s}}{\Gamma\left(m_{m}\right) \Gamma\left(m_{s}\right) \Omega_{0}}\right) \\
& \times H_{0,2}^{2,0}\left[\left(\frac{m_{m} m_{s}}{\Omega_{0}}\right) x \mid\left(m_{m}-1,1\right),\left(m_{s}-1,1\right)\right], x>0 .
\end{aligned}
$$

Now by applying [15, Eq. (6.2.8)], the expression in (3) follows.

Further, substituting (3) in [16, Eq. (26)] and utilizing [15, Eq. (6.2.4)], the CDF of GK can be written as

$$
\begin{aligned}
& P_{\gamma}(\gamma)=\frac{1}{\Gamma\left(m_{m}\right) \Gamma\left(m_{s}\right)} \\
& \times G_{1,3}^{2,1}\left[\left(\frac{m_{m} m_{s}}{\Omega_{0}}\right) \gamma \mid \begin{array}{l}
1 \\
m_{m}, m_{s}, 0
\end{array}\right], \gamma>0,
\end{aligned}
$$

where $G[\cdot]$ is the Meijer G-function.

\section{BER ANALYSIS}

In SC combining scheme, the highest SNR branch is selected. In our case, for dual-diversity, the SNR, $\gamma_{s c}$, is given by

$$
\gamma_{s c}=\max \left(\gamma_{1}, \gamma_{2}\right) .
$$

The CDF of $\gamma_{s c}$ is given by

$$
F\left(\gamma_{s c}\right)=\operatorname{Pr}\left(\max \left(\gamma_{1}, \gamma_{2}\right) \leq \gamma_{s c}\right)=\prod_{n=1}^{2} F_{\gamma_{n}}\left(\gamma_{s c}\right) .
$$

The BER for SC is given by

$P_{e}=\int_{0}^{\infty} P_{e}\left(\epsilon \mid \gamma_{s c}\right) f_{\gamma_{s c}}(\gamma) d \gamma_{s c}=\int_{0}^{\infty} P_{e}\left(\epsilon \mid \gamma_{s c}\right) d F\left(\gamma_{s c}\right)$,

where $P_{e}\left(\epsilon \mid \gamma_{s c}\right)$ is the conditional error probability (CEP) for the given SNR. A unified CEP expression for coherent and non-coherent binary modulation schemes over an AWGN channel is given in [17] as

$$
P_{e}\left(\epsilon \mid \gamma_{s c}\right)=\frac{\Gamma\left(p, q \gamma_{s c}\right)}{2 \Gamma(p)},
$$

where $\Gamma(\cdot, \cdot)$ is the complementary incomplete gamma function [14, Eq. (8.350.2)]. The parameters $p$ and $q$ in (10) account for different modulation schemes. For an extensive list of modulation schemes represented by these parameters, one may look into [18]. Now, applying integration by parts in (9), we get

$$
P_{e}=\left.P_{e}\left(\epsilon \mid \gamma_{s c}\right) F\left(\gamma_{s c}\right)\right|_{0} ^{\infty}-\int_{0}^{\infty} F\left(\gamma_{s c}\right) d P_{e}\left(\epsilon \mid \gamma_{s c}\right) .
$$

The first term goes to zero using [19, Eq. (6.5.3)]. Further, substituting (10) into (11) and using [19, Eq. (6.5.25)], the average BER can be written as

$$
P_{e}=\frac{q^{p}}{2 \Gamma(p)} \int_{0}^{\infty} \exp \left(-q \gamma_{s c}\right) \gamma_{s c}^{p-1} F\left(\gamma_{s c}\right) d \gamma_{s c} .
$$


Substituting (8) in the above obtained expression, we get

$$
P_{e}=\frac{q^{p}}{2 \Gamma(p)} \int_{0}^{\infty} \exp \left(-q \gamma_{s c}\right) \gamma_{s c}^{p-1} \prod_{n=1}^{2} F_{\gamma_{n}}(\gamma) d \gamma_{s c}
$$

Note that the integrand in (13) involves the product of the two independent Meijer $G$-functions which can be expressed in terms of extended generalized bivariate Meijer $G$-function (EGBMGF) which was introduced in [20] and whose Mathematica ${ }^{\circledR}$ implementation is given in Table II of [21].

Using [20], we obtain the product of the CDFs present in the above expression in terms of EGBMGF as

$$
\begin{aligned}
& \prod_{n=1}^{2} F_{\gamma_{n}}(\gamma)=F_{\gamma_{1}}(\gamma) F_{\gamma_{2}}(\gamma)= \\
& \left.\kappa_{1} S\left[\begin{array}{l}
0,0 \\
0,0
\end{array}\right] \mid \begin{array}{l}
1,2 \\
0,1 \\
1,2 \\
0,1
\end{array}\right) \\
&
\end{aligned}
$$

where $S[\cdot]$ is the EGBMGF as given in [20, Eq. (2.1)], $\kappa_{1}=\frac{1}{\Gamma\left(m_{m_{1}}\right) \Gamma\left(m_{s_{1}}\right) \Gamma\left(m_{m_{2}}\right) \Gamma\left(m_{s_{2}}\right)}, \kappa_{2}=m_{m_{1}}, m_{s_{1}}, 0, \kappa_{3}=$ $m_{m_{2}}, m_{s_{2}}, 0, \kappa_{4}=\frac{m_{m_{1} m_{s_{1}}}}{\Omega_{o_{1}}}$, and $\kappa_{5}=\frac{m_{m_{2}} m_{s_{2}}}{\Omega_{o_{2}}}$.

Now, substituting (14) into (13), then using the useful identity presented in [20, Eq. (2.1)] and then performing additional manipulations, we get the desired closed-form expression for the average BER as

$$
\left.P_{e}=\frac{\kappa_{1}}{2 \Gamma(p)} S\left[\begin{array}{l}
1,0 \\
0,0 \\
1,2 \\
0,1 \\
1,2 \\
0,1
\end{array}\right) \quad \begin{array}{c|c} 
& \\
1 ; \kappa_{2} & \frac{\left(\kappa_{4}\right)}{q} \\
1 ; \kappa_{3} & \frac{\left(\kappa_{5}\right)}{q} \\
&
\end{array}\right] .
$$

\section{RESUlTS AND DisCUSSION}

The numerical results for BER of SC scheme with dualdiversity over i.n.i.d. GK fading channels are presented in this section.

To our best knowledge, the EGBMGF is not available in standard mathematical packages. As such, we use our own efficient Mathematica ${ }^{\circledR}$ implementation of this function given in Table II of [21] in order to offer numerical results based on (15). With this implementation, the EGBMGF can be evaluated fast and accurately. This computability, therefore, has been utilized for different digital modulation schemes and is employed to discuss the results in comparison to respective Monte Carlo simulation outcomes.

The average SNR per bit in all the scenarios discussed is assumed to be equal. In addition, different digital modulation schemes are represented based on the values of $p$ and $q$ where $p=0.5$ and $q=1$ represents binary phase shift keying (BPSK), $p=1$ and $q=1$ represents differential phase shift keying (DPSK) and binary frequency shift keying (BFSK) is represented by $p=0.5$ and $q=0.5$. In Monte Carlo simulations, the GK fading channel was generated by the product of two independent gamma RVs.

We observe from Fig. 1 that this implemented computability of EGBMGF provides a perfect match to the MATLAB simulated results and the results are as expected i.e. the BER increases as the shadowing effect increases (i.e. value of $m_{s}$ decreases) while keeping multipath fading constant at $m_{m}=1$. The figure shown here represents BPSK. Its important to note here that these values for the parameters were selected randomly to prove the validity of the obtained results and hence specific values based on the standards can be used to obtain the required results.

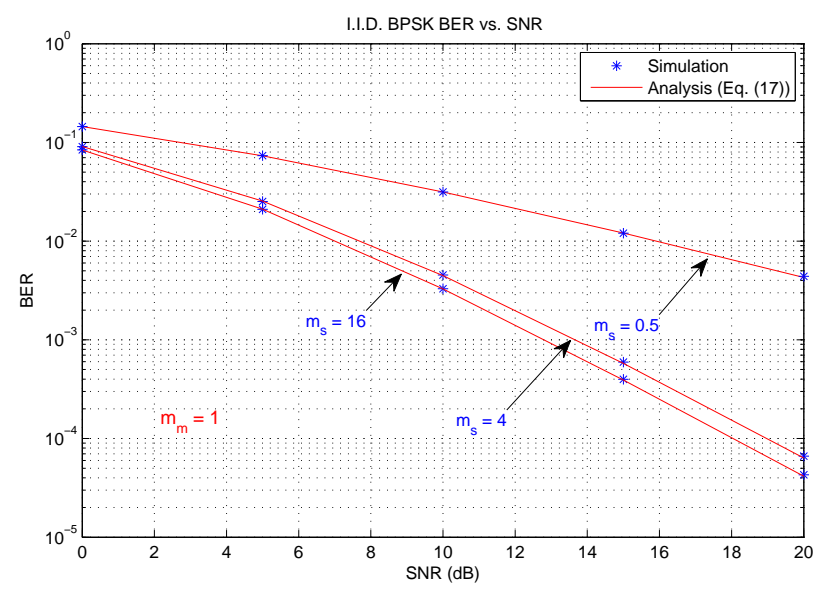

Fig. 1. Average BER of BPSK over i.i.d. GK fading channels with dualbranch SC $\left(m_{m}=1\right.$ and varying $\left.m_{s}\right)$.

Furthermore, to demonstrate the case that the results presented in this section also handle the presence of i.n.i.d. GK channels, following Fig. 2 presents the different modulation schemes with different effects of multipath fading and shadowing on both their channels. The values utilized for multipath fading and shadowing were as follows; $m_{m_{1}}=1, m_{m_{2}}=2$, $m_{s_{1}}=0.5$, and $m_{s_{2}}=4$. It can be seen that, as expected, BPSK outperforms the other modulation schemes and BFSK and DPSK perform in similar fashion at lower SNR whereas as the SNR increases DPSK performs better than BFSK.

Similar results for any other values of $m_{m}^{\prime} s$ and $m_{s}^{\prime} s$ can be observed for the exact closed-form BER for dual-diversity i.n.i.d. GK channels presented in this work.

\section{CONCLuding REMARKS}

An exact closed-form expression for the BER performance of different binary modulations with dual-branch SC scheme over i.n.i.d. GK fading was derived. The analytical calculations were done utilizing a general class of special functions, specifically, the EGBMGF. In addition, this work presents numerical examples to illustrate the mathematical formulation developed in this work and to show the effect of the fading and shadowing severity and unbalance on the system performance. 


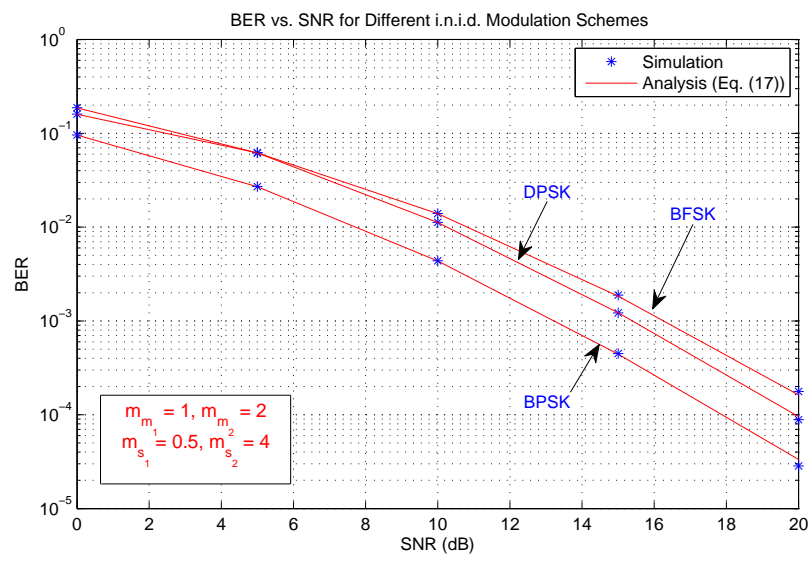

Fig. 2. BER for different modulation schemes undergoing i.n.i.d. channels with $m_{m_{1}}=1, m_{m_{2}}=2, m_{s_{1}}=0.5$, and $m_{s_{2}}=4$.

\section{ACKNOWLEDGMENT}

We would like to thank King Abdullah University of Science and Technology (KAUST), Thuwal, Makkah Province, Saudi Arabia, and Carleton University, Ottawa, Canada for providing support and resources respectively for this research work.

\section{REFERENCES}

[1] M. K. Simon and M.-S. Alouini, Digital Communication over Fading Channels, 2nd ed. Hoboken, New Jersey, USA: IEEE John Wiley \& Sons, Inc., 2005.

[2] H.-C. Yang, "New results on ordered statistics and analysis of minimumselection generalized selection combining (GSC)," IEEE Transactions on Wireless Communications, vol. 5, no. 7, pp. 1876-1885, July 2006.

[3] Y.-C. Ko, M.-S. Alouini, and M. K. Simon, "Analysis and optimization of switched diversity systems," IEEE Transactions on Vehicular Technology, vol. 49, no. 5, pp. 1813-1831, Sep. 2000.

[4] D. J. Lewinsky, "Nonstationary probabilistic target and clutter scattering models," IEEE Transactions on Antenna and Propagation, vol. AP-31, no. 3, pp. 490-498, May 1983.

[5] P. M. Kostic, "Analytical approach to performance analysis for channel subject to shadowing and fading," IEE Proceedings on Communications, vol. 152 , no. 6, pp. 821-827, Dec. 2005.

[6] P. M. Shankar, "Error rates in generalized shadowed fading channels," Wireless Personal Communications, vol. 28, no. 4, pp. 233-238, Feb. 2004.

[7] P. S. Bithas, N. C. Sagias, P. T. Mathiopoulos, G. K. Karagiannidis, and A. A. Rontogiannis, "On the performance analysis of digital communications over generalized- $K$ fading channels," IEEE Communication Letters, vol. 5, no. 10, pp. 353-355, May 2006.

[8] S. Al-Ahmadi and H. Yanikomeroglu, "On the approximation of the generalized- $K$ distribution by a Gamma distribution for modeling composite fading channels," IEEE Transactions on Wireless Communications, vol. 9, no. 2, pp. 706-713, Feb. 2010.

[9] M.-S. Alouini and M. K. Simon, "Dual diversity over correlated lognormal fading channels," IEEE Transactions on Communications, vol. 50, pp. 1946-1959, 2002.

[10] M. H. Ismail and M. M. Matalgah, "Bit error rate of diversity $M$-phaseshift keying receivers in Weibull fading with cochannel interference," IET Communications, vol. 4, iss. 1, pp. 13-25, 2010.

[11] P. M. Shankar, "Performance analysis of diversity combining algorithms in shadowed fading channels," Wireless Personal Communications, vol. 37, no. 1-2, pp. 61-72, Apr. 2006.

[12] P. S. Bithas, P. T. Mathiopoulos and S. A. Kotsopoulos, "Diversity reception over generalized- $K\left(K_{G}\right)$ fading channels," IEEE Transactions on Wireless Communications, vol. 6, no. 12, pp. 4238-4243, Dec. 2007.
[13] J. M. Romero-Jerez and A. J. Goldsmith, "Performance of multichannel reception with transmit antenna selection in arbitrarily distributed Nakagami fading channels," IEEE Transactions on Wireless Communications, vol. 8, no. 4, pp. 2006-2013, Apr. 2009.

[14] I. S. Gradshteyn and I. M. Ryzhik, Table of Integrals, Series and Products. New York: Academic Press, 2000.

[15] M. D. Springer, The Algebra of Random Variables. John Wiley Sons, Inc., 1979.

[16] V. S. Adamchik and O. I. Marichev, "The algorithm for calculating integrals of hypergeometric type functions and its realization in reduce system," Proceedings of the International Conference/Symposium on Symbolic and Algebraic Computation, pp. 212-224, 1990.

[17] A. H. Wojnar, "Unknown bounds on performance in Nakagami channels," IEEE Transactions on Communications, vol. 34, no. 1, pp. 22-24, Jan. 1986.

[18] N. C. Sagias, D. A. Zogas, and G. K. Kariaginnidis, "Selection diversity receivers over nonidentical Weibull fading channels," IEEE Transactions on Vehicular Technology, vol. 54, no. 6, pp. 2146-2151, Nov. 2005.

[19] M. Abramowitz and I. A. Stegun, Handbook of Mathematical Functions, New York: Dover, 1972.

[20] M. Shah, "On generalization of some results and their applications," Collectanea Mathematica, vol. 24, no. 3, pp. 249-266, 1973.

[21] I. S. Ansari, S. Al-Ahmadi, F. Yilmaz, M.-S. Alouini, and H. Yanikomeroglu, "A new formula for the BER of binary modulations with dual-branch selection over generalized- $K$ composite fading channels," submitted to IEEE Transactions on Communications. Available in arxiv.org at http://arxiv.org/abs/1012.3788. 